\title{
Estimates for Difference Quotients of Solutions of Poisson Type Difference Equations*
}

\author{
By Achi Brandt $\dagger$
}

1. Introduction. The following problem is frequently treated in the literature: Given a finite-difference operator $L$ defined for a function $\phi$ on some open, connected region $R$, with boundary $\dot{R}$, what estimates can be given for $\sup _{R}|\phi|$ in terms of $\sup _{R}|L \boldsymbol{\phi}|$ and $\sup _{R}|\phi|$ ? Such estimates are essential for the appraisal of discretization and round-off errors in a finite-difference approximation to the solution of a differential equation. For a linear operator $L$ of nonnegative type (i.e. $L$ satisfies a maximum principle), estimates of this kind are derivable by the well-known Gerschgorin [6] method and its extensions (see [5]). The method is to bound $|\phi|$ by a certain comparison function $\Psi$, by showing, using the maximum principle, that both $\phi-\Psi \leqq 0$ and $-\phi-\Psi \leqq 0$ throughout the region.

However, there is practically nothing so far published concerning the more delicate problem of estimating difference-quotients of the function $\phi$ in terms of $\sup _{R}|\boldsymbol{L} \phi|$ and the boundary data. Such estimates are necessary for the assessment of discretization and round-off errors introduced whenever a derivative of the solution of a differential equation is computed from the finite-difference approximation. These estimates are also indispensable, in some (especially nonlinear) cases, for the proof of the convergence of the numerical scheme itself. (See for example in D. F. DeSanto and HI. B. Keller [3], where such estimates for the Laplace operator are needed to demonstrate the convergence of a numerical scheme representing some incompressible viscous flow.) We believe such estimates might be useful for various existence proofs associated with nonlinear finite-difference equations.

The purpose of this paper is, therefore, to describe a method which gives bounds for the difference-quotients, up to the second order, of a discrete function $\phi$, the bounds depending on $\sup _{R}|L \phi|$, where $L$ is of the nonnegative type, and on some boundary conditions. The boundary conditions are, of course, of crucial importance here, since for points near the boundary the difference-quotients of $\phi$ depend heavily on the smoothness of $\phi$ on the boundary, as well as on the smoothness of the boundary itself. Of course, for points away from the boundary this dependence on the smoothness of the boundary conditions disappears, and we can estimate the difference-quotients in terms of $\sup _{R}|L \phi|$ and $\sup _{\dot{R}}|\phi|$ alone. Estimates which are confined to an interior region bounded away from the boundary, are called "interior estimates".

Some typical interior estimates obtained in this paper are the following:

THEOREM 1.1. In an n-dimensional Euclidean space, let $R$ be the open cube with the origin $\mathbf{0}=(0,0, \cdots, 0)$ at its center, and with side length $2 b$; i.e.

$$
R=\left\{\left(x_{1}, x_{2}, \cdots, x_{n}\right)|| x_{k} \mid<b, 1 \leqq k \leqq n\right\} ;
$$

Received May 26, 1966.

* This work represents results obtained at the Courant Institute of Mathematical Sciences, New York University, under a National Science Foundation Grant No. NSF GP-3465.

$\dagger$ The author is on leave from the Weizmann Institute, Rehoveth, Israel. 
and let $\dot{R}$ be its boundary. If then

$$
|\Delta \phi| \leqq \delta \text { in } R
$$

and

$$
|\phi| \leqq \mu \text { on } \dot{R}
$$

then

$$
\begin{array}{rlrl}
|\phi(0)| & \leqq \mu+\frac{1}{2} b^{2} \delta, & r \\
\left|\frac{\partial}{\partial x_{k}} \phi(0)\right| & \leqq \frac{n}{b} \mu+\frac{1}{2} b \delta, & 1 \leqq k \leqq n, \\
\left|\frac{\partial^{2}}{\partial x_{k} \partial x_{l}} \phi(0)\right| & \leqq \frac{4 n}{b^{2}} \mu+\left(\frac{2}{3} \log \frac{b}{h}\right) \delta, & 1 \leqq k<l \leqq n, \\
\left|\frac{\partial^{2}}{\partial x_{k}^{2}} \phi(0)\right| & \leqq \frac{4(n-1)}{b^{2}} \mu & \\
+\left[\frac{1}{n}+\frac{4}{3}\left(1-\frac{1}{n}\right) \log \frac{b}{h}\right] \delta, & 1 \leqq k \leqq n .
\end{array}
$$

Here $\Delta$ and $\partial$ are not the usual Laplace and partial-differentiation operators, respectively, but rather they represent difference-quotients analogous to them, on a square lattice with mesh spacing $h$.

This theorem, which we prove in Section 3, provides estimates for differencequotients not only at the center of a cube, as stated, but actually (as explained at the beginning of Section 5) it yields interior estimates for practically any region. Also we show that, under conditions (1.2) and (1.3), inequalities (1.4)-(1.7) are, in fact, the best possible estimates, except possibly for some minor improvements in the numerical coefficients.

Although we restrict our proof to the finite-difference formulation of Theorem 1.1 , it should be noted that the differential analogue of this theorem (as well as of any other hereingiven) is also true, and basically the same proofs are applicable. By the "differential analogue of Theorem 1.1" we mean, of course, that the $\Delta$ and $\partial$ operators have their usual differential meaning. However, this cannot be the meaning of $\partial$ in estimates like (1.6) or (1.7), where explicit dependence on the mesh-size $h$ is exhibited. For the continuous analogues of these estimates, the operator $\partial^{2} / \partial x_{k} \partial x_{l}$ in (1.6) (and similarly $\partial^{2} / \partial x_{k}{ }^{2}$ ) should be interpreted as a first-order differencequotient of a first-order partial derivative, notwithstanding the interpretation of $\Delta$ as the differential Laplacian. This leads, by corresponding changes in the proofs, to the estimate

$$
\text { (1.6a) } \frac{1}{2 h}\left|\frac{\partial}{\partial x_{l}} \phi\left(h e_{k}\right)-\frac{\partial}{\partial x_{l}} \phi\left(-h e_{k}\right)\right| \leqq \frac{4 n}{b^{2}} \mu+\left(\frac{2}{3} \log \frac{2 b}{h}\right) \delta, \quad 1 \leqq l \neq k \leqq n,
$$

where $e_{k}$ is the unit vector in the $x_{k}$ direction.

In contrast to the absence of published material about the finite difference case, there is a vast literature concerning estimates for the derivatives of solutions of partial differential equations. (See [7].) J. Schauder [8], [9] obtained such estimates, bounding the modulus of any first- or second-order derivative of the solution $\phi$, for 
any linear elliptic equations of the second order $L \phi=f$. He obtained both interior estimates and estimates near the boundary. The former depend on $\sup |\phi|$, sup $|f|$, and the Hölder-continuity of $f$ and of the coefficients of $L$, while the latter estimates also depend on Hölder-norms related to the smoothness of the boundary conditions. Motivated by Schauder estimates, we show, in Section 4 , that also in the finite difference case, if $\Delta \phi$ satisfies a Hölder-condition at a point, then the second-order difference-quotients at that point have bounds not depending on $h$.

Schauder estimates were extended to elliptic systems of great generality by $\mathbf{A}$. Douglis and L. Nirenberg [4] and by S. Agmon, A. Douglis and L. Nirenberg [1], [2]. In addition, the latter gave analogous $L_{p}$ estimates, for $p>1$, up to the boundary. They also present an extensive bibliography on this subject. The differential analogies to most of our theorems are just special cases of the results of this extensive literature. Only inequalities (1.6), or (1.6a), and (1.7) seem to have no counterpart therein. The methods we use to obtain these results are quite different from those used in the above theory. The proofs in the above mentioned papers rely on potential theoretic considerations, which, presumably, cannot be conveniently translated into a discrete form. (This may explain the sparsity of literature on the finite-difference case. A work by H. Montvila [8], whose methods are essentially discrete analogues to the usual continuous methods, comes out with estimates much weaker than ours.) Our methods are more elementary, using only the maximum principle and some symmetries exhibited by the operators. Thus, incidentally, the continuous analogues of our proofs provide more elementary derivations of some of the known differential results.

Our results and proofs are themselves capable of generalization in several directions. In Section 5 we discuss the continuation of the interior estimates to the vicinity of some straight portion of the boundary. For more general boundaries similar methods are applicable, with some additional complexities common to all finite-difference calculations near curved boundaries. This work is currently in progress.

In Section 6 the up-to-the-boundary estimates for the Dirichlet problem for the Laplacian are generalized to the discrete version of the operator

$$
L=\Delta+p(x, y) \partial / \partial x+q(x, y) \partial / \partial y,
$$

which, for constant $p$ and $q$, is essentially the canonical form of the elliptic equation of second order with constant coefficients. Generalization of the estimates to other discrete elliptic operators are at present under investigation.

In Section 7 we present up-to-the-boundary estimates for the Neumann problem, to illustrate how our techniques have to be modified in this case. In a subsequent paper we plan to present some of the above-indicated generalizations.

The author is indebted to Professor Louis Nirenberg for helpful discussions.

2. Preliminary Notation and Lemmas. The methods used in this and the forthcoming sections are applicable for Euclidean spaces of arbitrary dimension (higher than 1). However, for convenience of notation, we shall regularly restrict ourselves to the $x-y$ plane, leaving for later remarks the slight modifications necessary for higher dimensions.

For the finite-difference formulation we cover the plane by a square lattice of net 
points $P_{i, j}=\left(x_{i}, y_{j}\right)$, with

$$
x_{i}=i h, \quad y_{j}=j h,
$$

where $i$ and $j$ are integers and $h$ some fixed positive constant (the mesh spacing). In the present paper we consider some open, connected region whose boundary is solely composed of vertical and horizontal links of the net, so that the region is actually a union of meshes of the net. The set of net points which are interior to that region is called $R$, and the set of net points which lie on its boundary is called $\dot{R}$. It is such a set of net points $R$ that we mean hereinafter when we speak of "a region". We also regularly denote $\bar{R}=R+\dot{R}$.

The special types of regions, rectangles and infinite strips, which we oonsider are denoted respectively by

$$
R_{a b}=\left\{\left(x_{i}, y_{j}\right)|| x_{i}|<a,| y_{j} \mid<b\right\}
$$

and

$$
R_{b}=\left\{\left(x_{i}, y_{j}\right)|| y_{j} \mid<b\right\} .
$$

It is always assumed in this paper that $a=M h, b=N h$, where $M$ and $N$ are positive integers. By the above convention we now also have

$$
\begin{aligned}
\bar{R}_{a b} & =\left\{\left(x_{i}, y_{j}\right)|| x_{i}|\leqq a,| y_{j} \mid \leqq b\right\}, \\
\bar{R}_{b} & =\left\{\left(x_{i}, y_{j}\right)|| y_{j} \mid \leqq b\right\},
\end{aligned}
$$

and

$$
\begin{aligned}
\dot{R}_{a b} & =\bar{R}_{a b}-R_{a b}, \\
\dot{R}_{b} & =\bar{R}_{b}-R_{b} .
\end{aligned}
$$

The following definition of "distance along the lattice" will be useful:

$$
d(P, Q)=\left|x_{P}-x_{Q}\right|+\left|y_{P}-y_{Q}\right|,
$$

where $P=\left(x_{P}, y_{P}\right)$ and $Q=\left(x_{Q}, y_{Q}\right)$ are any two lattice points. For any set $S$ of lattice points we put

$$
d(P, S)=\min _{Q \in S} d(P, Q) .
$$

For any function $\phi$ defined on the mesh we employ the notation

$$
\begin{aligned}
\phi_{i, j} & =\phi\left(x_{i}, y_{j}\right)=\phi\left(P_{i, j}\right), \\
\partial_{x} \phi_{i, j} & =(1 / 2 h)\left(\phi_{i+1, j}-\phi_{i-1, j}\right), \\
\partial_{y} \phi_{i, j} & =(1 / 2 h)\left(\phi_{i, j+1}-\phi_{i, j-1}\right), \\
\partial_{x y} \phi_{i, j} & =\left(1 / 4 h^{2}\right)\left(\phi_{i+1, j+1}+\phi_{i-1, j-1}-\phi_{i+1, j-1}-\phi_{i-1, j+1}\right), \\
\partial_{x x} \phi_{i, j} & =\left(1 / h^{2}\right)\left(\phi_{i+1, j}+\phi_{i-1, j}-2 \phi_{i, j}\right), \\
\partial_{y y} \phi_{i, j} & =\left(1 / h^{2}\right)\left(\phi_{i, j+1}+\phi_{i, j-1}-2 \phi_{i, j}\right), \\
\Delta \phi_{i, j} & =\left(1 / h^{2}\right)\left(\phi_{i+1, j}+\phi_{i, j+1}+\phi_{i-1, j}+\phi_{i, j-1}-4 \phi_{i, j}\right) .
\end{aligned}
$$


Notice that $\partial_{x}, \partial_{y}$ and $\partial_{x y}$ are defined by central difference expressions. For treatment of other possible definitions, see Section 8.

We shall use the discrete analogue of the maximum principle in the following form:

Lemma 2.1. If $R$ is a region for which

$$
\sup _{\boldsymbol{P} \in R} d(P, \dot{R})<\infty
$$

and if $\phi$ is a bounded function for which ${ }^{1}$

$$
\Delta \phi(R) \leqq 0
$$

and

$$
\phi(\dot{R}) \geqq 0
$$

then

$$
\phi(R) \geqq 0 .
$$

Note that condition (2.1) is obviously satisfied whenever $R$ is a bounded region, and in this case the boundedness of $\phi$ should not be explicitly required. But condition (2.1) is also satisfied in other cases, e.g. any region $R$ which is a subset of the infinite strip $R_{b}$.

Proof. We put

$$
N h=\sup _{P \in R} d(P, \dot{R})
$$

and

$$
\mu=\inf _{R} \phi .
$$

This means that, for any $\epsilon>0$, there exist a point $P_{0}=\left(x_{I}, y_{J}\right)$ in $R$ such that

$$
\phi_{I, J}<\mu+\epsilon .
$$

By (2.2), (2.6) and (2.7) there follows

$$
\begin{aligned}
\phi_{I, J+1} & \leqq 4 \phi_{I, J}-\phi_{I-1, J}-\phi_{I+1, J}-\phi_{I, J-1} \\
& <4(\mu+\epsilon)-3 \mu=\mu+4 \epsilon,
\end{aligned}
$$

similar inequalities being satisfied by $\phi_{I, J-1}, \phi_{I+1, J}$ and $\phi_{I-1, J}$. Repeating this argument $N$ times one gets, for each point $P_{N}$ such that $d\left(P_{0}, P_{N}\right)=N h$, that

$$
\phi\left(P_{N}\right)<\mu+4^{N} \epsilon .
$$

Now by (2.5) there indeed exists a point $P_{N} \in \dot{R}$ with $d\left(P_{0}, P_{N}\right)=N h$. Thus (2.5) and $(2.9)$, together with $(2.3)$, lead to

$$
\mu+4^{N} \epsilon>0 .
$$

This, being true for any positive $\epsilon$, implies $\mu \geqq 0$, as claimed in (2.4).

${ }^{1}$ This is a convenient abbreviation for $\Delta \phi_{i, j} \leqq 0$ for any $\left(x_{i}, y_{j}\right) \in R$. Such obvious abbreviations are repeatedly used below. 
Substituting $\phi-\Psi$ for $\phi$ in Lemma 2.1, we get the more general

Lemia 2.2. If the region $R$ fulfills (2.1) and if $\phi$ and $\Psi$ are bounded and

$$
\Delta \phi_{i, j} \leqq \Delta \Psi_{i, j}, \quad\left(x_{i}, y_{j}\right) \in R,
$$

and

$$
\phi_{i, j} \geqq \Psi_{i, j}, \quad\left(x_{i}, y_{j}\right) \in \dot{R},
$$

then

$$
\phi_{i, j} \geqq \Psi_{i, j}, \quad\left(x_{i}, y_{j}\right) \in R .
$$

An immediate corollary of this is

Lemma 2.3. If $R \subseteq R_{b}$, and if $\phi$ is bounded and

$$
\begin{gathered}
-\delta_{2} \leqq \Delta \phi(R) \leqq-\delta_{1}, \\
\mu_{2} \geqq \phi(\dot{R}) \geqq \mu_{1},
\end{gathered}
$$

then

$$
\mu_{2}+\left(\delta_{2} / 2\right)\left(b^{2}-y_{j}{ }^{2}\right) \geqq \phi_{i, j} \geqq \mu_{1}+\left(\delta_{1} / 2\right)\left(b^{2}-y_{j}{ }^{2}\right), \quad\left(x_{i}, y_{j}\right) \in R .
$$

It is also easily deduced from Lemma 2.2 that the Dirichlet problem, for the discrete Poisson equation in a region $R$ which satisfies (2.1), has one and only one bounded solution.

3. Interior Estimates for the Dirichlet-Poisson Discrete Operator. In this section difference-quotients of a function $\phi$ at the center of a square $R_{b b}$ are estimated in terms of $\Delta \phi\left(R_{b b}\right)$ and $\phi\left(\dot{R}_{b b}\right)$. This square $R_{b b}$ is shown in Fig. 1, where a notation is introduced for some points related to that square. Reference to this notation is made in subsequent proofs, in an obvious manner. Thus, $R_{A B C D}$ designates the (discrete) interior part of the rectangle $A B C D ; \dot{R}_{A B C D}$ designates the (discrete) boundary of that rectangle; $A B$ is simply the segment $A B$; etc.

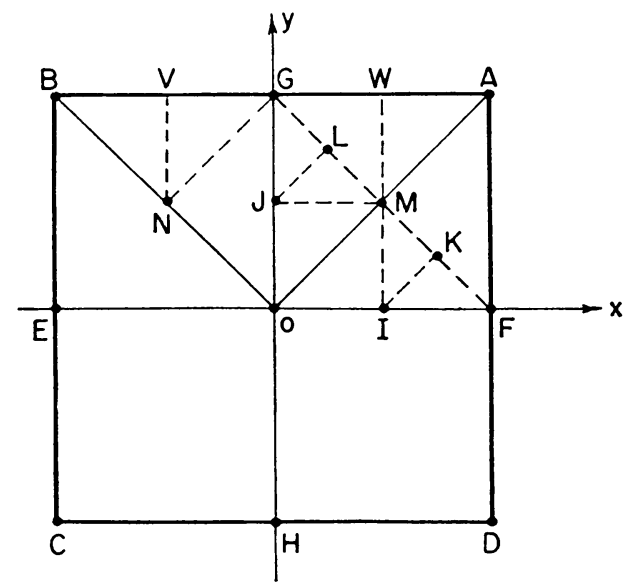

FIGURE 1. The square $\bar{R}_{b b}=\bar{R}_{A B C D}$ and auxiliary notation. The denoted points have coordinates as follows: $A(b, b), B(-b, b), C(-b,-b), D(b,-b), E(-b, 0), F(b, 0), G(0, b)$, $H(0,-b), I\left(\frac{1}{2} b, 0\right), J\left(0, \frac{1}{2} b\right), K\left(\frac{3}{4} b, \frac{1}{4} b\right), L\left(\frac{1}{4} b, \frac{3}{4} b\right), M\left(\frac{1}{2} b, \frac{1}{2} b\right), N\left(-\frac{1}{2} b, \frac{1}{2} b\right), O(0,0), V\left(-\frac{1}{2} b, b\right)$, $W\left(\frac{1}{2} b, b\right)$. 


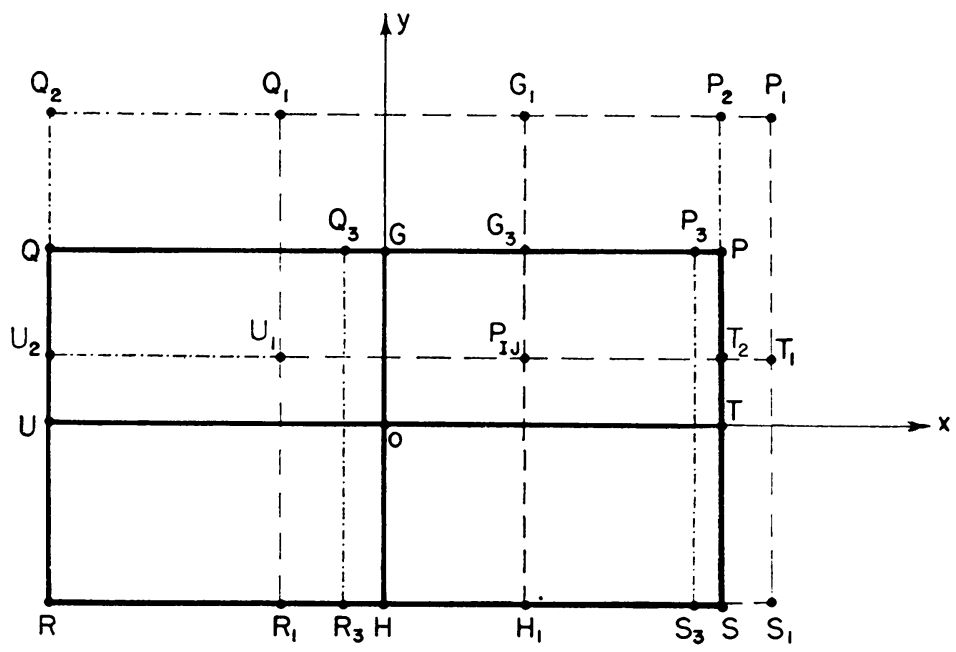

Figure 2. The rectangle $\bar{R}_{a b}=\bar{R}_{P Q R S}$ and auxiliary notalion. The points have coordinates as follows: $G(0, b), H(0,-b), O(0,0), P(a, b), Q(-a, b), R(-a,-b), S(a,-b), T(a, 0), U(-a, 0)$. $P_{I J}$ is the arbitrary net point (of Section 5 ) whose (positive) coordinates are $\left(x_{I}, y_{J}\right)$, where $x_{I}<a, y_{J}<b$. This point determines the location of all the subscripted points by requiring that $\bar{R}_{P_{1} Q_{1} R_{1} S_{1}}$ be a square with base on the line $R S$ and center at $P_{I J} ; T_{2} U_{2}$ be a centerline, through $P_{I J}$, of the rectangle $\bar{R}_{P_{2} Q_{2} R S}$; and $G_{3} H_{1}$ is a centerline, through $P_{I J}$, of the square $\vec{R}_{P_{3} Q_{3} R_{3} S_{3}}$.

The first two lemmas of this section have slightly more general forms than necessary for the interior estimates (Theorem 3.1). Instead of the square $R_{b b}$, these lemmas deal with the rectangle $R_{a b}$, and refer therefore to the notation introduced in Fig. 2. The more general forms of these lemmas will be useful in Section 5 .

LEMMA 3.1. If

$$
\phi\left(\dot{R}_{a b}\right)=0
$$

and.

$$
\left|\Delta \phi\left(R_{a b}\right)\right| \leqq \delta
$$

then

$$
\left|\partial_{y} \phi_{i, 0}\right| \leqq b \delta / 2
$$

for every $\left|x_{i}\right| \leqq a$.

Proof. We define the antisymmetric function

$$
\Psi_{i, j}=\frac{1}{2}\left(\phi_{i, j}-\phi_{i,-j}\right) .
$$

This gives

$$
\begin{aligned}
\Psi_{i, 1} & =h \partial_{y} \phi_{i, 0} \\
\left|\Delta \Psi_{i, j}\right| & \leqq \frac{1}{2}\left(\left|\Delta \phi_{i, j}\right|+\left|\Delta \phi_{i,-j}\right|\right) \leqq \delta, \quad\left(x_{i}, y_{j}\right) \in R_{b b}
\end{aligned}
$$

and

$$
\Psi\left(\dot{R}_{P Q U T}\right)=0 .
$$


By Lemma 2.2 it now follows from (3.6) and (3.7) that

$$
\left|\Psi_{i, j}\right| \leqq \frac{1}{2} y_{i}\left(b-y_{i}\right) \delta, \quad\left(x_{i}, y_{j}\right) \in R_{P Q U T},
$$

and in particular

$$
\left|\Psi_{i, 1}\right| \leqq \frac{1}{2} h(b-h) \delta,
$$$$
\left|x_{i}\right| \leqq a,
$$

which, together with (3.5)), involves (3.3).

Lemia 3.2. If

$$
\begin{aligned}
\Delta \phi\left(R_{a b}\right) & =0, \\
\phi(Q R) & =\phi(R S)=\phi(S P)=0
\end{aligned}
$$

and

$$
|\phi(P Q)| \leqq \mu
$$

then

$$
\left|\partial_{y} \phi_{i 0}\right| \leqq \mu / 2 b
$$

for each $\left|x_{i}\right| \leqq a$.

Proof. Again we define $\Psi$ to be the antisymmetric part of $\phi$, as in (3.4). In the present case this entails (3.5) as well as

$$
\begin{aligned}
\Delta \Psi\left(R_{a b}\right) & =0, \\
\Psi(Q U) & =\Psi(U T)=\Psi(T P)=0,
\end{aligned}
$$

and

$$
|\Psi(P Q)| \leqq \frac{1}{2} \mu .
$$

By Lemma 2.2 applied to $R_{P Q U T}$ we therefore have

$$
\left|\Psi_{i, j}\right| \leqq \mu y_{j} / 2 b, \quad\left(x_{i}, y_{j}\right) \in R_{P Q U T},
$$

so that, by (3.5),

$$
\left|\partial_{y} \phi_{i, 0}\right|=(1 / h)\left|\Psi_{i, 1}\right| \leqq \mu / 2 b .
$$

We could of course interchange $P Q$ with $R S$ in the above lemma. The next lemma is of the same type, but its proof is more involved and gives an estimate at the center of the square $R_{b b}$ only. (Notation thus refers to Fig. 1.) ${ }^{2}$

Lemia 3.3. If

$$
\begin{aligned}
\Delta \phi\left(R_{b b}\right) & =0, \\
\phi(A B) & =\phi(B C)=\phi(C D)=0,
\end{aligned}
$$

and

$$
|\phi(D A)| \leqq \mu,
$$

then

$$
\left|\partial_{y} \phi_{0,0}\right| \leqq \mu / 2 b .
$$

\footnotetext{
${ }^{2}$ Simpler and better proofs to Lemmas 3.3, 3.6 and 3.7 are included in a subsequent paper.
} 
Proof. The auxiliary function $\Psi$ is defined by

$$
\Psi_{i, j}=\frac{1}{4}\left(\phi_{i, j}-\phi_{i,-j}+\phi_{-i, j}-\phi_{-i,-j}\right)
$$

which entails

$$
\begin{aligned}
\Psi_{0,1} & =h \partial_{y} \phi_{0,0}, \\
\Delta \Psi\left(R_{b b}\right) & =0, \\
\Psi(A B) & =\Psi(E F)=0,
\end{aligned}
$$

and

$$
|\Psi(A F)| \leqq \frac{1}{2} \mu, \quad|\Psi(B E)| \leqq \frac{1}{2} \mu .
$$

We further define three other auxiliary functions, $\Psi^{1}, \Psi^{2}$ and $\Psi^{3}$, by requiring

$$
\begin{aligned}
\Psi^{1}(E F) & =0, \quad \Psi^{1}(B E)=\Psi^{1}(A F)=-\frac{1}{2} \mu, \quad \Psi^{1}(A B)=\frac{1}{2} \mu, \\
\Delta \Psi^{1}\left(R_{A B E F}\right) & =0 ; \\
\Psi^{2}(E F) & =0, \quad \Psi^{2}(B E)=\Psi^{2}(A F)=-\frac{1}{2} \mu, \quad \Psi^{2}(A B)=\frac{1}{2} \mu, \\
\Psi^{2}(O G) & =0, \quad \Delta \Psi^{2}\left(R_{A G O F}\right)=\Delta \Psi^{2}\left(R_{G B E O}\right)=0 ; \\
\Psi^{3}(E F) & =\Psi^{3}(A B)=0, \quad \Psi^{3}(B E)=\Psi^{3}(A F)=\frac{1}{2} \mu, \\
\Delta \Psi^{3}\left(R_{A B E F}\right) & =0 .
\end{aligned}
$$

Clearly, $\Psi^{2}$ in $\bar{R}_{A G O F}$ is antisymmetric about the diagonal $O A$ (its symmetric part vanishing on $\dot{R}_{A G O F}$ and therefore also throughout $\left.R_{A G O F}\right)$. Thus $\Psi^{2}(O A)=0$, and (assuming $\mu>0$ )

$$
\Psi^{2}\left(R_{O A G}\right)>0
$$

Similarly

$$
\Psi^{2}\left(R_{O B G}\right)>0 .
$$

This means that $\Psi^{2}$ has no local maximum at any interior point of the segment $G O$. At other points of $R_{A B E F}$ we have defined $\Psi^{2}$ to be discrete harmonic, so that $\Psi^{2}$ has no local maximum throughout $R_{A B E F}$. Now $\Psi^{1}$ is discrete harmonic anywhere in $R_{A B E F}$. Thus $\Psi^{2}-\Psi^{1}$ also cannot have any local maximum in $R_{A B E F}$. But on the boundary $\dot{R}_{A B E F}$ the function $\Psi^{2}-\Psi^{1}$ vanishes, and so we have proved that

$$
\Psi_{i, j}^{2}-\Psi_{i, j}^{1} \leqq 0
$$

$$
\left(x_{i}, y_{j}\right) \in R_{A B E F} .
$$

This, together with (3.29) and (3.30), give

$$
\Psi^{1}\left(\bar{R}_{O B A}\right) \geqq 0 .
$$

Using Lemma 2.2 we gather, from (3.23)-(3.26) and (3.28), the following two inequalities:

$$
\begin{array}{rlrl}
\left|\Psi_{i, j}\right| & \leqq \Psi_{i, j}^{3}, & \left(x_{i}, y_{j}\right) & \in R_{A B E F}, \\
\Psi_{i, j}^{3}+\Psi_{i, j}^{1} & \leqq \mu y_{j} / 2 b, & \left(x_{i}, y_{j}\right) \in R_{A B E F} .
\end{array}
$$


These inequalities, along with (3.32), entail

$$
\left|\Psi_{i, j}\right| \leqq \mu y_{j} / 2 b, \quad\left(x_{i}, y_{j}\right) \in \bar{R}_{A O B},
$$

which gives, by $(3.22 \mathrm{~b})$, the desired estimate.

The next lemmas estimate second-order difference-quotients. Again we shall use "antisymmetrization" methods, except that now "double-antisymmetrization" will be used, instead of the "one-direction antisymmetrization" employed for the above first-order estimates.

Lemia 3.4. If

$$
\phi\left(\dot{R}_{b b}\right)=0
$$

and

$$
\left|\Delta \phi\left(R_{b b}\right)\right| \leqq \delta
$$

then

$$
\left|\partial_{x y} \phi_{0,0}\right| \leqq\left(\frac{2}{3} \log (b / h)\right) \delta .
$$

Proof. We define the auxiliary doubly-antisymmetric function

$$
\Psi_{i, j}=\frac{1}{4}\left(\phi_{i, j}+\phi_{-i,-j}-\phi_{i,-j}-\phi_{-i, j}\right) .
$$

This function clearly satisfies

$$
\begin{array}{r}
\Psi\left(\dot{R}_{A G O F}\right)=0 \\
\left|\Delta \Psi\left(R_{A B C D}\right)\right| \leqq \delta
\end{array}
$$

and

$$
\Psi_{1,1}=h^{2} \partial_{x y} \phi_{0,0} .
$$

To estimate $\Psi$ we introduce the comparison function

$$
\chi(x, y)=x y \log [2 b /(x+y)], \quad x \geqq 0, y \geqq 0,
$$

for which the following interesting properties are easily verified:

$$
\begin{aligned}
\chi\left(\dot{R}_{A G O F}\right) & \geqq 0, \\
-2 & \leqq \Delta \chi\left(R_{A G O F}\right) \leqq-\frac{3}{2} .
\end{aligned}
$$

By Lemma 2.2, applied to $R_{A G O F}$, we therefore get

$$
\left|\Psi_{i, j}\right| \leqq \frac{2}{3} \delta \chi\left(x_{i}, y_{j}\right), \quad\left(x_{i}, y_{j}\right) \in R_{A G O F},
$$

and in particular

$$
\left|\Psi_{1,1}\right| \leqq \frac{2}{3} \delta \chi(h, h)=\frac{2}{3} \delta h^{2} \log (b / h),
$$

so that, in virtue of (3.42), our lemma is proved.

LeMma 3.5. If $\phi$ satisfies (3.36) and (3.37) then

$$
\max \left(\left|\partial_{x x} \phi_{0,0}\right|,\left|\partial_{y y} \phi_{0,0}\right|\right) \leqq\left(\frac{1}{2}+\frac{2}{3} \log (b / h)\right) \delta .
$$

Proof. We follow here practically the same lines as in the proof of Lemma 3.4, except that the antisymmetrizations are done with respect to the lines $x= \pm y$ 
rather than" the axis. That is, here we put

$$
\Psi_{i, j}=\frac{\mathbf{1}}{4}\left(\phi_{i, j}+\phi_{-i,-j}-\phi_{j, i}-\phi_{-j,-i}\right)
$$

which again provides (3.41), and also yields

$$
\Psi\left(\dot{R}_{B O A}\right)=0,
$$

and

$$
\Psi_{0,1}=\left(h^{2} / 4\right)\left(\partial_{y y} \phi_{0,0}-\partial_{x x} \phi_{0,0}\right) .
$$

The comparison function in the present case is defined as

$$
\chi(x, y)=\frac{1}{2}\left(y^{2}-x^{2}\right) \log (b / y),
$$$$
y \geqq|x|,
$$

which again has the property (3.45), and also

$$
\chi\left(\dot{R}_{B O A}\right)=0 .
$$

Thus, applying Lemma 2.2 to the triangle $R_{B O A}$, we get

$$
\left|\Psi_{i, j}\right| \leqq \frac{2}{3} \delta \chi\left(x_{i}, y_{j}\right)
$$$$
\left(x_{i}, y_{j}\right) \in R_{B O A}
$$

and in particular

$$
\left|\Psi_{0,1}\right| \leqq \frac{2}{3} \delta \chi(0, h)=\frac{1}{3} \delta h^{2} \log (b / h) .
$$

This estimate, together with (3.51) and the requirement (3.37), yields (3.48).

Lemma 3.6. If

$$
\Delta \phi\left(R_{b b}\right)=0
$$

and

$$
\left|\phi\left(\dot{R}_{b b}\right)\right| \leqq \mu
$$

then

$$
\left|\partial_{x y} \phi_{0,0}\right| \leqq 8 \mu / b^{2}
$$

Proof. We define $\Psi$ as in (3.39), so that (3.42) holds. Also, by (3.56) and (3.57),

$$
\begin{array}{r}
\Delta \Psi\left(R_{A B C D}\right)=0, \\
\left|\Psi\left(\dot{R}_{A B C D}\right)\right| \leqq \mu
\end{array}
$$

and

$$
\Psi(E F)=\Psi(O G)=0 .
$$

Four other auxiliary functions, $\Psi^{0}, \Psi^{1}, \Psi^{2}$ and $\Psi^{3}$, are defined as follows:

$$
\begin{array}{rlrl}
\Psi_{i, j}^{0} & =8 \mu x_{i} y_{j} / b^{2}, & & \left(x_{i}, y_{j}\right) \in R_{G O F} \\
\Psi^{1}(G O) & =\Psi^{1}(O F)=0, & & \Psi^{1}(G F)=\mu, \quad \Delta \Psi^{1}\left(R_{G O F}\right)=0 ; \\
\Psi^{2}(G O) & =\Psi^{2}(O F)=0, & & \Psi^{2}(G M)=(4 x / b-1) \mu \\
\Psi^{2}(M F) & =(4 y / b-1) \mu, & \Delta \Psi^{2}\left(R_{G O F}\right)=0 ; \\
\Psi^{3}(G O) & =\Psi^{3}(O F)=0, & \Psi^{3}\left(\bar{R}_{M J O I}\right)=0, & \Psi^{3}(G M)=(4 x / b-1) \mu \\
\Psi^{3}(M F) & =(4 y / b-1) \mu, & \Delta \Psi^{3}\left(R_{G J M}\right)=\Delta \Psi^{3}\left(R_{M I F}\right)=0 .
\end{array}
$$


On $M F$ the function $\Psi^{3}$ is linear and vanishes at $K$. Hence, in the triangle $R_{M I F}$, $\Psi^{3}$ is antisymmetric about the median $I K$, and so (assuming $\mu>0$ )

$$
\Psi^{3}\left(R_{M I K}\right)>0 .
$$

Similiarly

$$
\Psi^{3}\left(R_{M J L}\right)>0 .
$$

The function $\Psi^{3}$ has therefore no maximum in $R_{G O F}$. The discrete harmonic function $\Psi^{2}$ has no interior minimum and so the difference $\Psi^{3}-\Psi^{2}$ cannot have any maximum in $R_{G O F}$. But on the boundary $\dot{R}_{G O F}$ this difference $\Psi^{3}-\Psi^{2}$ vanishes, and thus it should be nonpositive throughout $R_{G O F}$, i.e.

$$
\Psi_{i, j}^{3} \leqq \Psi_{i, j}^{2}, \quad\left(x_{i}, y_{j}\right) \in R_{G O \boldsymbol{F}} .
$$

By (3.65), (3.66), (3.67) and (3.68) we finally have

$$
\Psi^{2}\left(R_{\text {LJOIK }}\right) \geqq 0 \text {, }
$$

which is the main point of this proof. By using Lemma 2.2 for $R_{\text {GOF }}$ we get the following two inequalities:

$$
\begin{array}{rlrl}
\left|\Psi_{i, j}\right| & \leqq \Psi_{i, j}^{1}, & & \left(x_{i}, y_{j}\right) \in R_{\text {GOF }}, \\
\Psi_{i, j}^{1}+\Psi_{i, j}^{2} \leqq \Psi_{i, j}^{0}, & & \left(x_{i}, y_{j}\right) \in R_{\text {GOF }} .
\end{array}
$$

The inequalities (3.69), (3.70) and (3.71) entail

$$
\left|\Psi_{i, j}\right| \leqq \Psi_{i, j}^{0}, \quad\left(x_{i}, y_{j}\right) \in R_{\text {LJOIK }}
$$

In particular

$$
\left|\Psi_{1,1}\right| \leqq \Psi_{1,1}^{0}=8 \mu h^{2} / b^{2}
$$

which, in virtue of (3.42), proves our lemma.

Lemma 3.7. If $\phi$ satisfies (3.56) and (3.57), then

$$
\max \left(\left|\partial_{x x} \phi_{0,0}\right|,\left|\partial_{y y} \phi_{0,0}\right|\right) \leqq 4 \mu / b^{2} .
$$

Proof. We define the doubly-antisymmetric function $\Psi$ as in (3.49), which, under the conditions of the present lemma, implies

$$
\begin{gathered}
\Delta \Psi\left(R_{A B C D}\right)=0, \\
|\Psi(A B)| \leqq \mu, \quad \Psi(O A)=\Psi(O B)=0,
\end{gathered}
$$

and by $(3.56)$,

$$
\left(2 / h^{2}\right) \Psi_{0,1}=\frac{1}{2}\left(\partial_{y y} \phi_{0,0}-\partial_{x x} \phi_{0,0}\right)=\partial_{y y} \phi_{0,0}=-\partial_{x x} \phi_{0,0} .
$$

Auxiliary functions are defined by

$$
\Psi_{1, j}^{0}=2 \mu\left(y_{j}^{2}-x_{i}^{2}\right) / b^{2},
$$

$$
\begin{array}{rlrl}
\Psi_{1, j}^{0} & =2 \mu\left(y_{j}{ }^{2}-x_{i}{ }^{2}\right) / b^{2}, & \left(x_{i}, y_{j}\right) \in \bar{R}_{A O B} \\
\Psi^{1}(O A) & =\Psi^{1}(O B)=0, & \Psi^{1}(A B)=\mu, & \Delta \Psi^{1}\left(R_{A O B}\right)=0 \\
\Psi^{2}(O A) & =\Psi^{2}(O B)=0, & \Psi^{2}(A B)=(1-2|x| / b) \mu, & \Delta \Psi^{2}\left(R_{A O B}\right)=0 .
\end{array}
$$

In the same way as (3.69) is established in the proof of Lemma 3.6, it is here shown 
that

$$
\Psi^{2}\left(R_{V N O M W}\right) \geqq 0 .
$$

Lemma 2.2, applied here to the triangle $R_{A O B}$, leads to the two inequalities

$$
\begin{aligned}
\left|\Psi_{i, j}\right| & \leqq \Psi_{i, j}^{1}, & \left(x_{i}, y_{j}\right) & \in R_{A O B}, \\
\Psi_{i, j}^{1}+\Psi_{i, j}^{2} & \leqq \Psi_{i, j}^{0}, & \left(x_{i}, y_{j}\right) & \in R_{A O B},
\end{aligned}
$$

which, together with (3.81), involve

$$
\left|\Psi_{i, j}\right| \leqq \Psi_{i, j}^{0}, \quad\left(x_{i}, y_{j}\right) \in R_{V_{N O M W}}
$$

This, by (3.77), completes the proof.

THEOREM 3.1. If

$$
\left|\Delta \phi\left(R_{b b}\right)\right| \leqq \delta
$$

and

$$
\left|\phi\left(\dot{R}_{b b}\right)\right| \leqq \mu
$$

then

$$
\begin{aligned}
\left|\phi_{0,0}\right| & \leqq \mu+\frac{1}{2} b^{2} \delta, \\
\max \left(\left|\partial_{x} \phi_{0,0}\right|,\left|\partial_{y} \phi_{0,0}\right|\right) & \leqq 2 \mu / b+\frac{1}{2} b \delta, \\
\left|\partial_{x y} \phi_{0,0}\right| & \leqq 8 \mu / b^{2}+\left(\frac{2}{3} \log (b / h)\right) \delta,
\end{aligned}
$$

and

$$
\max \left(\left|\partial_{x x} \phi_{0,0}\right|,\left|\partial_{y y} \phi_{0,0}\right|\right) \leqq 4 \mu / b^{2}+\left(\frac{1}{2}+\frac{2}{3} \log (b / h)\right) \delta .
$$

Proof. By the linearity of the Dirichlet-Laplace operator, this theorem is a direct corollary of Lemmas 2.3, 3.1-3.7.

Remark A. By symmetry, $\mu$ in estimates (3.87)-(3.90) may be replaced by

$$
\mu^{\prime}=\frac{1}{8}\left(\mu_{A G}+\mu_{G B}+\mu_{B E}+\mu_{E C}+\mu_{C H}+\mu_{H D}+\mu_{D F}+\mu_{F A}\right),
$$

where $(3.86)$ is replaced by

$$
|\phi(A G)| \leqq \mu_{A G}, \quad|\phi(G B)| \leqq \mu_{G B}, \quad \text { etc. }
$$

Remark B. With slight modifications, all the above lemmas and proofs are applicable for functions of $n$ variables, with $\Delta$ defined as the $n$-dimensional discrete Laplace operator. Practically no change is needed in Lemmas 2.3, 3.1-3.4 and their proofs, and the changes to be introduced in the other lemmas are quite obvious in nature. As a result, the following generalization of Theorem 3.1 is obtained:

Theorem 3.2. In n-dimensional Euclidean space with coordinates $x_{1}, x_{2}, \cdots x_{n}$ let $h$ be some positive increment and $N$ some positive integer, and $b=N h$; and let

$$
\begin{aligned}
& R_{b(n)}=\left\{x_{k}=i_{k} h \mid i_{k} \text { integer, }\left|i_{k}\right|<N,(1 \leqq k \leqq n)\right\}, \\
& \bar{R}_{b(n)}=\left\{x_{k}=i_{k} h \mid i_{k} \text { integer },\left|i_{k}\right| \leqq N,(1 \leqq k \leqq n)\right\}, \\
& \dot{R}_{b(n)}=\bar{R}_{b(n)}-R_{b(n)} .
\end{aligned}
$$


With this notation, if

$$
\left|\Delta \phi\left(R_{b(n)}\right)\right| \leqq \delta
$$

and

$$
\left|\phi\left(\dot{R}_{b(n)}\right)\right| \leqq \mu
$$

then

$$
\begin{array}{rlr}
\left|\phi_{0}\right| & \leqq \mu+\frac{1}{2} b^{2} \delta \\
\left|\partial_{x_{k}} \phi_{0}\right| & \leqq n \mu / b+\frac{1}{2} b \delta, & 1 \leqq k \leqq n \\
\left|\partial_{x_{k} x_{l}} \phi_{0}\right| & \leqq 4 n \mu / b^{2}+\left(\frac{2}{3} \log (b / h)\right) \delta, & 1 \leqq k<l \leqq n
\end{array}
$$

and

$$
\left|\partial_{x_{k} x_{k}} \phi_{0}\right| \leqq 4(n-1) \mu / b^{2}+\left[1 / n+\frac{4}{3}(1-1 / n) \log (b / h)\right] \delta, \quad 1 \leqq k \leqq n .
$$

Here the operators $\Delta$ and $\partial$ denote the $n$-dimensional analogues to the central difference-quotients defined in (2.0). The subscript 0 denotes the origin $(0,0$, $\cdots, 0$ ), which is at the center of the cube $R_{b(n)}$.

Remark on possible improvements to the estimates. Under the conditions there imposed, all the above estimates are the best possible ones, except for a possible reduction of the numerical coefficients. The lowest possible coefficients can be easily computed numerically.

For example, we show this for the estimate of Lemma 3.4. Let $\bar{\phi}$ be the function for which

$$
\begin{aligned}
\bar{\phi}\left(\dot{R}_{b b}\right) & =\bar{\phi}(E F)=\bar{\phi}(G H)=0, \\
\Delta \bar{\phi}\left(R_{A G O F}\right) & =\Delta \bar{\phi}\left(R_{C H O E}\right)=\delta,
\end{aligned}
$$

and

$$
\Delta \bar{\phi}\left(R_{B E O G}\right)=\Delta \bar{\phi}\left(R_{D F O H}\right)=-\delta .
$$

It is clear by symmetry that

$$
\Delta \bar{\phi}(E F)=\Delta \bar{\phi}(G H)=0,
$$

so that $\bar{\phi}$ satisfies the conditions (3.36) and (3.37). Furthermore, if $\phi$ is any other function satisfying these conditions, we may again define $\Psi$ as in (3.39), and then show by (3.40), (3.41), (3.42) and Lemma 2.2 that

$$
\left|\partial_{x y} \phi_{0,0}\right| \leqq \partial_{x y} \bar{\phi}_{0,0} \text {. }
$$

Thus, by numerically solving $(3.100)-(3.102)$ we would get the best coefficient to replace the $\frac{2}{3}$ in estimate (3.38). This coefficient cannot be less than $\frac{1}{2}$, since, in virtue of the left-side inequality in (3.45), it is inferable by Lemma 2.2 that

$$
\bar{\phi}_{i, j} \geqq \frac{1}{2} \delta x_{i} y_{j} \log \left[b /\left(x_{i}+y_{j}\right)\right], \quad\left(x_{i}, y_{j}\right) \in R_{A G O F},
$$

so that

$$
\partial_{x y} \bar{\phi}_{0,0} \geqq \frac{1}{2} \delta \log (b / 2 h) .
$$


This example obviously shows that the dependence on $|\log h|$ exhibited in Theorems 3.1 and 3.2 cannot be suppressed, unless more restrictive conditions are specified. Theorems with such extra restrictions are the objective of the next section.

4. Interior Estimates for Hölder Continuous $\Delta \phi$. For the differential case, it is known [7] that when $\Delta \phi$ satisfies a Hölder condition, such a condition is obtainable also for every second-order derivative of $\phi$. Motivated by this are the following lemmas and theorem, which give $h$-free estimates for the second-order differencequotients.

LEMMA 4.1. If

$$
\phi\left(\dot{R}_{b b}\right)=0
$$

and

$$
\left|\Delta \phi_{i, j}-\Delta \phi_{0,0}\right| \leqq H\left(\left|x_{i}\right|+\left|y_{j}\right|\right)^{\alpha}, \quad\left(x_{i}, y_{j}\right) \in R_{b b},
$$

for some constants $H$ and $0<\alpha<1$, then

$$
\left|\partial_{x y} \phi_{0,0}\right| \leqq 4 H b^{\alpha} / 3 \alpha \text {. }
$$

Proof. We define the auxiliary function $\Psi$ as in (3.39). The proof then follows roughly the same lines as that of Lemma 3.4, except that the comparison function for the present case should be

$$
\chi(x, y)=x y\left[(2 b)^{\alpha}-(x+y)^{\alpha}\right], \quad x \geqq 0, y \geqq 0 .
$$

This is a proper comparison function here, since it has the property

$$
-2 \leqq \Delta \chi(x, y) / \alpha(x+y)^{\alpha} \leqq-\frac{3}{2}, \quad x \geqq 0, y \geqq 0,
$$

and it also fulfills (3.44).

Remark. The condition (4.2) may be replaced by the weaker one

$$
\frac{1}{4}\left|\Delta \phi_{i, j}+\Delta \phi_{-i,-j}-\Delta \phi_{-i, j}-\Delta \phi_{i,-j}\right| \leqq H\left(\left|x_{i}\right|+\left|y_{j}\right|\right)^{\alpha}, \quad\left(x_{i}, y_{j}\right) \in R_{b b},
$$

which is all we need for the above proof.

LeMma 4.2. If $\phi$ satisfies conditions (4.1) and (4.2), then

$$
\max \left(\left|\partial_{x x} \phi_{0,0}\right|,\left|\partial_{y y} \phi_{0,0}\right|\right) \leqq 4 H b^{\alpha} / 3 \alpha+\frac{1}{2}\left|\Delta \phi_{0,0}\right| \text {. }
$$

Proof. We follow the method of proof of Lemma 3.5. As a comparison function we use here

$$
\chi(x, y)=\frac{1}{2}\left(y^{2}-x^{2}\right)\left[b^{\alpha}-y^{\alpha}\right], \quad y \geqq|x|,
$$

which fulfils (3.53), as well as

$$
-2 \leqq \Delta \chi(x, y) / \alpha y^{\alpha} \leqq-\frac{3}{2}, \quad y \geqq|x| .
$$

In the same way as in Section 3, these lemmas can be combined together with Lemmas 3.6 and 3.7, into one theorem:

Theorem 4.1. With constants $H$ and $0<\alpha<1$, if

$$
\left|\Delta \phi_{i, j}-\Delta \phi_{0,0}\right| \leqq H\left(\left|x_{i}\right|+\left|y_{j}\right|\right)^{\alpha}, \quad\left(x_{i}, y_{j}\right) \in R_{b b},
$$


and

$$
\left|\phi\left(\dot{R}_{b b}\right)\right| \leqq \mu
$$

then

$$
\left|\partial_{x y} \phi_{0,0}\right| \leqq 8 \mu / b^{2}+4 H b^{\alpha} / 3 \alpha
$$

and

$$
\max \left(\left|\partial_{x x} \phi_{0,0}\right|,\left|\partial_{y y} \phi_{0,0}\right|\right) \leqq 4 \mu / b^{2}+4 H b^{\alpha} / 3 \alpha+\frac{1}{2}\left|\Delta \phi_{0,0}\right| .
$$

Remark. As in Section 3 (in the remark following Theorem 3.2), it is here also easily demonstrated that, under conditions (4.9) and (4.10), estimates (4.11) and (4.12) are essentially the best possible ones.

It is known, however, that Hölder-continuity, like (4.9), is not necessary to get $h$-free estimates for the second order difference quotients. On the other hand, just continuity is not sufficient. This is shown by the example

$$
\phi(x, y)=x y \log \log \left[1 /\left(x^{2}+y^{2}\right)\right], \quad x^{2}+y^{2} \leqq e^{-1} .
$$

The Laplacian of this function is everywhere continuous, whereas its mixed secondorder derivative is not bounded, its leading term in the vicinity of $x=y=0$ being $\log \log \left[1 /\left(x^{2}+y^{2}\right)\right]$.

5. Estimates up to the Boundary. The theorems of Sections 3 and 4 provide estimates for the difference-quotients not only at the center of a square, but also, in fact, at any internal point of practically any region. Indeed, for each such internal point we may construct a square $S$, with boundary $\dot{S}$, completely contained in the region, with this point at its center. Now, a bound for $\sup _{\dot{s}}|\phi|$ is furnished by wellknown methods (Lemma 2.3, for instance) so that the above theorems are readily applicable, yielding estimates for the difference-quotients at the center of $S$, as desired.

However, in order to estimate the difference-quotients near the boundary of the region, account must obviously be taken of the smoothness of both the boundary data and the boundary itself. The simplest (but still typical) case is that of a straight portion of the boundary on which the function identically vanishes. In such a case we can simply use reflection to continue the function across this portion of the boundary. Some points in a neighborhood of the previous boundary now are bounded away from the new boundary, and can be handled by interior estimates.

As an example, we shall now use this method of reflection to get estimates for the difference-quotients everywhere in the rectangle $R_{a b}$.

THeorem 5.1. If

$$
\phi\left(\dot{R}_{a b}\right)=0
$$

and.

$$
\left|\Delta \phi\left(R_{a b}\right)\right| \leqq \delta,
$$

then, for any point $\left(x_{I}, y_{J}\right) \in R_{a b}$,

$$
\left|\partial_{y} \phi_{I, J}\right| \leqq b \delta
$$




$$
\begin{aligned}
\left|\partial_{x} \phi_{I, J}\right| & \leqq b \delta \\
\left|\partial_{x y} \phi_{I, J}\right| & \leqq\left(2+\frac{2}{3} \log (2 b / h)\right) \delta
\end{aligned}
$$

and

$$
\max \left(\left|\partial_{x x} \phi_{I, J}\right|,\left|\partial_{y y} \phi_{I, J}\right|\right) \leqq\left(\frac{3}{2}+\frac{2}{3} \log (2 b / h)\right) \delta .
$$

Proof. We first extend the domain of definition of $\phi$ from $\bar{R}_{a b}$ to the whole plane, by reflections across the boundary $\dot{R}_{a b}$, i.e., we require, for any $x$ and $y$ that are integral multiples of $h$,

$$
\begin{aligned}
\phi(a+x, y) & =-\phi(a-x, y), \\
\phi(-a+x, y) & =-\phi(-a-x, y), \\
\phi(x, b+y) & =-\phi(x, b-y),
\end{aligned}
$$

and

$$
\phi(x,-b+y)=-\phi(x,-b-y) .
$$

It is trivial that, for such a function $\phi$ that is given in $\bar{R}_{a b}$ and fulfills (5.1), these requirements indeed define a unique extension, to the entire plane. The condition (5.2) is now also extended to the whole plane

$$
\left|\Delta \phi_{i, j}\right| \leqq \delta
$$$$
-\infty<i, j<\infty
$$

Also, by Lemma 2.3,

$$
\left|\phi_{i, j}\right| \leqq \frac{1}{2} b^{2} \delta
$$$$
-\infty<i, j<\infty \text {. }
$$

Without loss of generality we may assume that $x_{I} \geqq 0, y_{J} \geqq 0$ and then employ the notation of Fig. 2. It is clear from (5.1) and (5.7)-(5.10) that

$$
\begin{gathered}
\phi\left(P_{2} S\right)=0, \\
\phi\left(P_{3} Q_{3}\right)=0, \\
\phi\left(Q_{2} R\right)=0,
\end{gathered}
$$

and

$$
\phi(R S)=\phi\left(R_{1} S_{1}\right)=\phi\left(R_{3} S_{3}\right)=0,
$$

and also, by (5.9) and Lemma 2.3,

$$
\left|\phi\left(P_{1} Q_{1}\right)\right|,\left|\phi\left(P_{2} Q_{2}\right)\right| \leqq \frac{1}{2} \delta\left[b^{2}-\left(b-2 y_{J}\right)^{2}\right]=2 \delta y_{J}\left(b-y_{J}\right) .
$$

From (5.11), (5.13), (5.15), (5.16) and (5.17) we get, through Lemmas 3.1 and 3.2 applied to the rectangle $R_{P_{2} Q_{2} R S}$, that

$$
\begin{aligned}
\left|\partial_{u} \phi_{I, J}\right| & \leqq \frac{2 \delta y_{J}\left(b-y_{J}\right)}{2\left(b+y_{J}\right)}+\frac{1}{2}\left(b+y_{J}\right) \delta \\
& =b \delta-\frac{\delta\left(b-y_{J}\right)^{2}}{2\left(b+y_{J}\right)} \\
& \leqq b \delta .
\end{aligned}
$$


Next, by considering Lemmas 3.1 and 3.2 on the square $R_{Q_{3} R_{3} S_{3} P_{3}}$, and taking into account (5.11), (5.12), (5.14) and (5.16), we obtain

$$
\left|\partial_{x} \phi_{I, J}\right| \leqq \frac{1}{2} b \delta+(1 / b) \frac{1}{2} b^{2} \delta=b \delta .
$$

Finally, we apply Theorem 3.1 (with Remark A that follows the theorem) to the square $R_{P_{1} Q_{1} R_{1} s_{1}}$, on the edges of which $|\phi|$ is bounded by $(5.16),(5.17)$ and (5.12), so as to get

$$
\begin{aligned}
\left|\partial_{x y} \phi_{I, J}\right| \leqq & \frac{8}{\left(b+y_{J}\right)^{2}} \cdot \frac{1}{4}\left(\delta b^{2}+2 \delta y_{J}\left(b-y_{J}\right)\right)+\left(\frac{2}{3} \log \frac{b+y_{J}}{h}\right) \delta \\
& =\left[2-6\left(y_{J} /\left(b+y_{J}\right)\right)^{2}+\frac{2}{3} \log \left[\left(b+y_{J}\right) / h\right] \delta\right. \\
& \leqq\left(2+\frac{2}{3} \log (2 b / h)\right) \delta
\end{aligned}
$$

as well as

$\max \left(\left|\partial_{x x} \phi_{I, J}\right|,\left|\partial_{y y} \phi_{I, J}\right|\right)$

$$
\begin{aligned}
& \leqq \frac{4}{\left(b+y_{J}\right)^{2}} \cdot \frac{1}{4}\left(\delta b^{2}+2 \delta y_{J}\left(b-y_{J}\right)\right)+\left(\frac{1}{2}+\frac{2}{3} \log \frac{b+y_{J}}{h}\right) \delta \\
& =\left[\frac{3}{2}-3\left(\frac{y_{J}}{b+y_{J}}\right)^{2}+\frac{2}{3} \log \frac{b+y_{J}}{h}\right] \delta \\
& \leqq\left(\frac{3}{2}+\frac{2}{3} \log (2 b / h)\right) \delta .
\end{aligned}
$$

Remark. All the estimates in Theorem 5.1 are intentionally written in terms of the length $b$ alone, without referring to the length $a$. By symmetry we can therefore replace $b$ in these estimates by $\min (a, b)$. Furthermore, the estimates are true for any $a$, no matter how large. In fact, they are true even for the case $a=\infty$, i.e. for the infinite strip $R_{b}$ (provided $\phi$ is not unbounded, cf. Section 2). The proof for this limiting case is altogether the same as the proof of Theorem 5.1.

Note that estimate (5.3) is precisely the best estimate for $\left|\partial_{y} \phi_{I, J}\right|$ not depending on the length $a$, the mesh size $h$ and the location $\left(x_{I}, y_{J}\right)$. Indeed, in the limiting case $a \rightarrow \infty, h \rightarrow 0$, the function

$$
\phi=\frac{1}{2}\left(b^{2}-y^{2}\right) \delta
$$

satisfies (5.1) and (5.2), and also exactly attains the bound of (5.3), since

$$
(\partial / \partial y) \phi(x, \pm b)=\mp b \delta .
$$

The method of extending interior estimates up to the boundary of a rectangle by means of reflections is also applicable to the $h$-free estimates of Section 4 . But in this case we have to impose some extra conditions in the vicinity of the corners of the rectangle. That such extra conditions are necessary we see by studying the function $\bar{\phi}$ defined in (3.100)-(3.102). This function apparently behaves very well in the square $R_{A G O F}$ : It has a constant $\Delta \bar{\phi}$ throughout this square, and on the boundary of this square $\bar{\phi}$ vanishes. Yet, it is clear from (3.105) that

$$
\partial_{x y} \bar{\phi}_{1,1} \geqq \frac{1}{2} \delta \log (b / 4 h),
$$

which means that in the neighborhood of a corner the mixed second-order difference- 
quotient is unbounded as $h \rightarrow 0$. This leads us to the introduction of condition

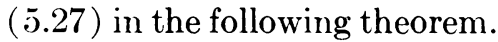

THEOREM 5.2. If

$$
\phi\left(\dot{R}_{a b}\right)=0,
$$

and, for some fixed point $\left(x_{I}, y_{J}\right) \in R_{a b}$ and fixed constants $H$ and $0<\alpha<1$,

$$
\left|\Delta \phi_{i, j}-\Delta \phi_{I, J}\right| \leqq H\left(\left|x_{i}-x_{I}\right|+\left|y_{j}-y_{J}\right|\right)^{\alpha}, \quad\left(x_{i}, y_{j}\right) \in R_{a b},
$$

and

$$
\left|\Delta \phi_{I, J}\right| \leqq H\left(a-\left|x_{I}\right|+b-\left|y_{J}\right|\right)^{\alpha},
$$

then

$$
\left|\partial_{x y} \phi_{I, J}\right| \leqq 7 H b^{\alpha} / 3 \alpha+8 \mu / b^{2}
$$

where

$$
\mu=\max _{R_{a b}}|\phi| \text {. }
$$

( $\mu$, in its turn, could be estimated by Lemma 2.3 or the like).

Proof. Again we extend the domain of definition of $\phi$ to the entire plane, by requiring (5.7)-(5.10). Let $i$ and $j$ be any two positive integers. By elementary geometrical considerations, conditions (5.26) and (5.27) yield

$$
\begin{aligned}
& \left|\Delta \phi_{I+i, J+j}+\Delta \phi_{I-i, J-j}-\Delta \phi_{I-i, J+j}-\Delta \phi_{I+i, J-j}\right| \\
& \leqq H\left\{4(A+B)^{\alpha}+\left(x_{i}+y_{j}\right)^{\alpha}+\left(\left|x_{i}-2 A\right|+y_{j}\right)^{\alpha}\right. \\
& \left.\quad+\left(x_{i}+\left|y_{j}-2 B\right|\right)^{\alpha}+\left(\left|x_{i}-2 A\right|+\left|y_{j}-2 B\right|\right)^{\alpha}\right\}
\end{aligned}
$$

where

$$
A=a-\left|x_{I}\right|, \quad B=b-\left|y_{J}\right| .
$$

For any positive $\xi, \eta$ and $\alpha$ it is true that $\xi^{\alpha}+\eta^{\alpha} \leqq 2(\xi+\eta)^{\alpha}$. Using this relation to estimate the right-hand side of (5.30), we derive

$$
\begin{array}{r}
\left|\Delta \phi_{I+i, J+j}+\Delta \phi_{I-i, J-j}-\Delta \phi_{I-i, J+j}-\Delta \phi_{I+i, J-j}\right| \leqq 7 H\left(x_{i}+y_{j}\right)^{\alpha} \\
0 \leqq x_{i}, y_{j} \leqq \infty .
\end{array}
$$

Hence, by Lemmas 4.1 (with the appended remark) and 3.6, we prove our theorem.

6. Estimates for $L=\Delta+p(x, y) \partial_{x}+q(x, y) \partial_{y}$. We define the finite-difference operator $L$ as follows:

$$
\begin{aligned}
L \phi_{i, j}= & \Delta \phi_{i, j}+p_{i, j} \partial_{x} \phi_{i, j}+q_{i, j} \partial_{y} \phi_{i, j} \\
= & h^{-2}\left[\left(1+h p_{i, j} / 2\right) \phi_{i+1, j}+\left(1-h p_{i, j} / 2\right) \phi_{i-1, j}\right. \\
& \left.\quad+\left(1+h q_{i, j} / 2\right) \phi_{i, j+1}+\left(1-h q_{i, j} / 2\right) \phi_{i, j-1}-4 \phi_{i, j}\right] .
\end{aligned}
$$

For constant $p$ and $q$, this operator is, basically, the canonical form of the second order elliptic equations with constant coefficients. Since we are primarily interested in estimates for the difference-quotients and not for the function itself, we do not 
give the slightly modified but technically cumbersome treatment that is required when we have an extra term $r_{i, j} \phi_{i, j}$ in $L$. In this section we give up-to-the-boundary estimates, in a rectangle, for $\phi$ and its difference-quotients, in terms of $\sup |L \phi|$ and the rectangle's width.

It is clear that no such estimates are possible unless we have some bounds for $p(x, y)$ and $q(x, y)$. We shall therefore assume throughout this section that finite bounds $P$ and $Q$ are given, such that

$$
\left|p_{i, j}\right| \leqq P, \quad\left|q_{i, j}\right| \leqq Q,
$$

wherever $L$ is defined. We shall also assume in this section that the mesh size $h$ is so small that

$$
h \leqq 2 \min \left(P^{-1}, Q^{-1}\right) .
$$

Under this condition $L$ is a nonnegative operator, i.e. all the parenthesized coefficients in the right-hand side of (6.1) are nonnegative, and therefore the following extension of Lemma 2.2 holds:

LEMMA 6.1. If the region $R$ satisfies (2.1) and (6.3), and if $\phi$ and $\Psi$ are bounded and

$$
L \phi_{i, j} \leqq L \Psi_{i, j}, \quad\left(x_{i}, y_{j}\right) \in R
$$

and

$$
\phi_{i, j} \geqq \Psi_{i, j}, \quad\left(x_{i}, y_{j}\right) \in \dot{R},
$$

then

$$
\phi_{i, j} \geqq \Psi_{i, j}, \quad\left(x_{i}, y_{j}\right) \in R .
$$

Proof. Owing to the linearity of $L$, it suffices to prove the lemma for $\Psi$ identically zero. For this case the proof is the same as the proof of Lemma 2.1, except that (2.2) is replaced by (6.4) and so (2.8) should be replaced by

$$
\begin{aligned}
\left(1+(h / 2) q_{I, J}\right) \phi_{I, J+1} \leqq & 4 \phi_{I, J}-\left(1-(h / 2) p_{I, J}\right) \phi_{I-1, J} \\
& -\left(1+(h / 2) p_{I, J}\right) \phi_{I+1, J}-\left(1-(h / 2) q_{I, J}\right) \phi_{I, J-1} \\
< & 4(\mu+\epsilon)-\left(1-(h / 2) p_{I, J}\right) \mu-\left(1+(h / 2) p_{I, J}\right) \mu \\
& -\left(1-(h / 2) q_{I, J}\right) \mu \\
= & \left(1+(h / 2) q_{I, J}\right) \mu+4 \epsilon,
\end{aligned}
$$

which implies

$$
\phi_{I, J+1}<\mu+K \epsilon
$$

where

$$
K=\max \left(\frac{4}{1-h P / 2}, \frac{4}{1-h Q / 2}\right) \text {. }
$$

This $K$ replaces the number 4 in (2.9) and (2.10), and the proof is completed in the same way. 
Lemma 6.2. If $R \subseteq R_{b}$ and

$$
h<\min \left(2 P^{-1}, Q^{-1}\right)
$$

and if $\phi$ is bounded and

$$
|L \phi(R)| \leqq \lambda,
$$

and

$$
|\phi(\dot{R})| \leqq \mu
$$

then

$$
\left|\phi_{i, j}\right| \leqq \mu+(\lambda / 2)\left(b^{2} e^{Q^{\prime} b}-y_{j}^{2} e^{Q^{\prime}\left|y_{j}\right|}\right), \quad\left(x_{i}, y_{j}\right) \in R,
$$

where $Q^{\prime}=11 Q / 10$. (For mesh size sufficiently small, e.g. $h \leqq 3\left(b^{-1} Q^{-3}\right)^{1 / 2}$, we can put $Q^{\prime}=Q$.)

Proof. We put $H=Q^{\prime} h$. By an elementary manipulation we get, for $y \geqq h$,

$$
\begin{aligned}
& L\left\{y^{2} e^{Q^{\prime}|y|}\right\} \\
& =e^{Q^{\prime} y}\left\{2+\left(y^{2}+h^{2}\right) Q^{\prime}\left[Q^{\prime} \frac{e^{H}+e^{-H}-2}{H^{2}}+q \frac{e^{H}-e^{-H}}{2 H}\right]\right. \\
& \left.+2 y\left[2 Q^{\prime} \frac{e^{H}-e^{-H}}{2 H}+q \frac{e^{H}+e^{-H}}{2}\right]\right\} \\
& \geqq e^{Q^{\prime} y}\left\{2+\left(y^{2}+h^{2}\right) Q^{\prime}\left[Q^{Q^{\prime}} \frac{e^{H}+e^{-H}-2}{\left.H^{2}-Q \frac{e^{H}-e^{-H}}{2 H}\right]}\right.\right. \\
& \left.+2 y\left[2 Q^{\prime} \frac{e^{H}-e^{-H}}{2 H}-Q \frac{e^{H}+e^{-H}}{2}\right]\right\} .
\end{aligned}
$$

By expanding $e^{H}$ and $e^{-H}$ in Taylor's series, bearing in mind (6.9b) which implies $H \leqq 1.1$, it is readily seen that, for $Q^{\prime}=11 Q / 10$, each of the two bracketed terms is positive. (For $Q^{\prime}=Q$ the first of these terms might be negative, but, for $h \leqq 3\left(b^{-1} Q^{-3}\right)^{1 / 2}$, the sum of the two terms is still positive.) Thus, for $y \geqq h$,

$$
L\left\{y^{2} e^{Q^{\prime}|y|}\right\} \geqq 2 e^{Q^{\prime} y} \geqq 2 .
$$

The same inequality is derived, in a similar way, for $y \leqq-h$, and also, in a trivial way, for $y=\mathbf{0}$. Consequently, for any $y$,

$$
L\left\{(\lambda / 2)\left(b^{2} e^{Q^{\prime} b}-y^{2} e^{Q^{\prime}|\boldsymbol{y}|}\right)\right\} \leqq-\lambda .
$$

The proof is now completed by Lemma 6.1, using (6.10), (6.11) and (6.15).

Lemma 6.3. If condition (6.3) is satisfied and if

$$
\begin{aligned}
\phi\left(\dot{R}_{a b}\right) & =0 \\
\left|L \phi\left(R_{a b}\right)\right| & \leqq \lambda
\end{aligned}
$$

and

$$
b(P+Q)<1
$$


then

$$
\left|\Delta \phi\left(R_{a b}\right)\right| \leqq \lambda /[1-b(P+Q)]
$$

and

$$
\max _{R_{a b}}\left(\left|\partial_{x} \phi_{i, j}\right|,\left|\partial_{y} \phi_{i, j}\right|\right) \leqq \lambda b /[1-b(P+Q)]
$$

Proof. We put

$$
\delta=\max _{R_{a b}}\left|\Delta \phi_{i, j}\right| .
$$

By (5.3) and (5.4) of Theorem 5.1 we have

$$
b \delta \geqq \max _{\boldsymbol{R}_{a b}}\left(\left|\partial_{x} \phi_{i, j}\right|,\left|\partial_{y} \phi_{i, j}\right|\right)=\eta,
$$

But, by (6.17) and (6.2) we also have

$$
\delta \leqq \lambda+\max _{\boldsymbol{R}_{a b}}\left|p_{i, j} \partial_{x} \phi_{i, j}+q_{i, j} \partial_{y} \phi_{i, j}\right| \leqq \lambda+(P+Q) \eta .
$$

The three inequalities (6.22), (6.23) and (6.18) immediately imply (6.19) and (6.20).

TheOReм 6.1. If

$$
\begin{array}{r}
\phi\left(\dot{R}_{a b}\right)=0, \\
\left|L \phi\left(R_{a b}\right)\right| \leqq \lambda,
\end{array}
$$

and

$$
h<(P+Q)^{-1}
$$

then

(i).2S) $\max _{R_{a b}}\left(\left|\partial_{x} \phi_{i, j}\right|,\left|\partial_{y} \phi_{i, j}\right|\right) \leqq \lambda b\left(\frac{1}{b(P+Q)}+\frac{2 b(P+Q)}{1-h(P+Q)} e^{Q^{\prime} b}\right)$

and

$$
\left|\Delta \phi\left(R_{a b}\right)\right| \leqq 2 \lambda\left(1+\frac{b^{2}(P+Q)^{2}}{1-h(P+Q)} e^{Q^{\prime b}}\right) .
$$

( $L, P, Q$ and $Q^{\prime}$ are defined in (6.1), (6.2) and Lemma 6.2.)

Remaiks. (A) For small $P+Q$, better estimates are given by Lemma 6.3 . (B) Estimate (6.29) provides, through Theorem 5, estimates for all the first and second order difference-quotients of $\phi$ in $R_{a b}$.

Proof. We define $\delta$ and $\eta$ as in (6.21) and (6.22), so that (6.23) holds. There exists, of course, a point $\left(x_{I}, y_{J}\right)$ in $R_{a b}$ such that

$$
\eta=\max \left(\left|\partial_{x} \phi_{I, J}\right|,\left|\partial_{y} \phi_{I, J}\right|\right) \text {. }
$$

For any length $c$ which is an integral multiple of $h$, we may construct a square of net points $\bar{S}_{c}$, with $\left(x_{I}, y_{J}\right)$ at its center and $2 c$ the length of its sides. If $\bar{S}_{c}$ is not contained in $\bar{R}_{a b}$, we extend the definition of $\phi$ to $\bar{S}_{c}$ by reflections, as in $(5.7)-(5.10)$. Consequently we have

$$
\left|\Delta \phi\left(S_{c}\right)\right| \leqq \delta
$$


and by Lemma 6.2 ,

$$
\left|\phi\left(\dot{S}_{c}\right)\right| \leqq(\lambda / 2) b^{2} e^{Q^{\prime} b}
$$

By Theorem 3.1 these inequalities entail

$$
\eta \leqq c \delta / 2+(2 / c)(\lambda / 2) b^{2} e^{Q^{\prime} b}
$$

This, together with (6.23), gives

$$
\eta \leqq(c / 2)[\lambda+\eta(P+Q)]+(\lambda / c) b^{2} e^{Q^{\prime} b} .
$$

Now, $(P+Q)^{-1}$ is not necessarily a multiple of $h$, but, by requirement $(6.27)$, we have some $0 \leqq \theta<1$ such that we may put

$$
c=(P+Q)^{-1}-\theta h .
$$

Then, in place of (6.34) we may write

$$
\eta \leqq \frac{1}{2} \lambda(P+Q)^{-1}+\frac{1}{2} \eta+\lambda b^{2}(P+Q) e^{Q^{\prime} b} /[1-h(P+Q)]
$$

which involves (6.28). And (6.29) then follows from (6.28) and (6.23).

7. Estimates for the Neumann Problem. Most of the estimates of Sections 3, 4, 5 and 6 have simple parallels in the case of Neumann boundary conditions, i.e. when the data are given in terms of $\partial_{n} \phi(\dot{R})$ instead of $\phi(\dot{R})$, where $\partial_{n}$ is some finite difference analogue to the normal derivative. In the present paper we are only interested in a rectangular region $R$, and the definition of $\partial_{n}$ is therefore quite straightforward. To each net point $P$ of the boundary $\dot{R}$ there is one and only one interior neighbour, $\hat{P}$ say, and we denote by $\check{P}$ an exterior neighbour of $P$ such that $P$ is the midpoint of the segment $\hat{P} \check{P}$. To be consistent with the definition (2.0) of $\partial_{x}$ and $\partial_{y}$, we define $\partial_{n}$ also as a central difference expression, namely

$$
\partial_{n} \phi(P)=(1 / 2 h)[\phi(P)-\phi(\hat{P})] .
$$

(Cf. Section 8 for a noncentral definition.) Note that (7.1) assumes $\phi$ to be defined also at the exterior point $\check{P}$, for each point $P$ in $\dot{R}$. Thus, to make the number of conditions equal the number of points, we should assume that $\Delta \phi$ is known not only in $R$ but also on the boundary $\dot{R}$.

Theorem 7.1. If

$$
\partial_{n} \phi\left(\dot{R}_{a b}\right)=0
$$

and

$$
\left|\Delta \phi\left(\bar{R}_{a b}\right)\right| \leqq \delta
$$

then

$$
\left|\partial_{y} \phi\left(\bar{R}_{a b}\right)\right| \leqq b \delta .
$$

Proof. We extend the definition of $\phi$ to the whole plane by requiring

$$
\begin{gathered}
\phi_{M+i, j}=\phi_{M-i, j}, \\
\phi_{-M+i, j}=\phi_{-M-i, j}, \\
\phi_{i, N+j}=\phi_{i, N-j},
\end{gathered}
$$


and

$$
\phi_{i,-N+j}=\phi_{i,-N-j},
$$

where $M=a / h, N=b / h$. By (7.2) and (7.3), these requirements uniquely define an extension, which satisfies, throughout the plane,

$$
\left|\Delta \phi_{i, j}\right| \leqq \delta
$$$$
-\infty<i, j<\infty
$$

We now introduce two antisymmetric functions

$$
\psi_{i, j}=\frac{1}{2}\left(\phi_{i, j}-\phi_{i,-j}\right), \quad-\infty<i, j<\infty,
$$

and

$$
\psi_{i, j}^{J}=\frac{1}{2}\left(\phi_{i, J+j}-\phi_{i, J-j}\right), \quad-\infty<i, j<\infty,
$$

where $J$ is any integer in the interval $0 \leqq J<N$. By (7.3)-(7.11) we clearly get

$$
\begin{aligned}
\psi_{i, 0} & =0, & -\infty<i<\infty, \\
\psi_{i, 0}^{J} & =0, & -\infty<i<\infty, \\
\left|\Delta \psi_{i, j}\right| & \leqq \delta, & -\infty<i, j<\infty,
\end{aligned}
$$

and

$$
\left|\Delta \psi_{i, j}^{J}\right| \leqq \delta, \quad-\infty<i, j<\infty
$$

Alse, by (7:10), (7.7) and (7.8),

$$
\psi_{i, 2 N}=\frac{1}{2}\left(\phi_{i, N+N}-\phi_{i,-N-N}\right)=\frac{1}{2}\left(\phi_{i, N-N}-\phi_{i,-N+N}\right)=0 .
$$

Thus, by (7.12), (7.14), (7.16) and Lemma 2.3 applied to the infinite strip $0 \leqq y \leqq 2 b$,

$$
\left|\psi_{i, N+j}\right| \leqq(\delta / 2)\left(b^{2}-y_{j}^{2}\right), \quad-b \leqq y_{j} \leqq b .
$$

('onsequently,

$$
\begin{aligned}
\left|\psi_{i, N}^{J}\right| & =\frac{1}{2}\left|\phi_{i, J+N}-\phi_{i, J-N}\right|=\frac{1}{2}\left|\phi_{i, N-J}-\phi_{i, J-N}\right| \\
& =\left|\psi_{i, N-J}\right| \leqq(\delta / 2)\left(b^{2}-y_{J}{ }^{2}\right) .
\end{aligned}
$$

This, together with (7.13), (7.15) and Lemma 2.2 applied to the strip $0 \leqq y_{j} \leqq b$, yield

$$
\left|\psi_{i, j}^{J}\right| \leqq \frac{\delta}{2} y_{j}\left(b-y_{j}\right)+\frac{1}{b} y_{j} \frac{\delta}{2}\left(b^{2}-y_{J}{ }^{2}\right), \quad 0 \leqq y_{j} \leqq b .
$$

Thus

$$
\left|\partial_{\nu} \phi_{i, J}\right|=\left|\frac{1}{h} \psi_{i, 1}^{J}\right| \leqq \frac{\delta}{2}(b-h)+\frac{\delta}{2 b}\left(b^{2}-y_{J}^{2}\right)<\delta b-\frac{\delta y_{J}{ }^{2}}{2 b} \leqq \delta b .
$$

Remark. This theorem clearly implies

$$
\left|\partial_{x} \phi\left(\bar{R}_{a b}\right)\right| \leqq a \delta .
$$

There is however no bound to $\partial_{x} \phi_{i, j}$ in terms of $b$ and $\delta$ alone, without referring to 
the length $a$. Indeed, the function

$$
\phi_{i, j}=\delta\left(a x_{i}-\left|x_{i}\right|\right)
$$

fulfills (7.2) and (7.3), but

$$
\partial_{x} \phi_{i, 0}=\delta\left(a-\frac{1}{2} h\right)
$$

is unbounded for indefinitely large $a$.

Theorem 7.2. If $\phi$ satisfies (7.2) and (7.3) then

$$
\left|\partial_{x y} \phi\left(\bar{R}_{a b}\right)\right| \leqq\left(8+\frac{2}{3} \log (b / h)\right) \delta
$$

and

$$
\max _{\bar{R}_{a b}}\left(\left|\partial_{x x} \phi_{i, j}\right|,\left|\partial_{y y} \phi_{i, j}\right|\right) \leqq\left(\frac{2 a}{b}+\frac{5}{2}+\frac{2}{3} \log \frac{b}{h}\right) \delta .
$$

Proof. We continue $\phi$ to the whole plane by requiring (7.5)-(7.8), which imply (7.9). For any point $P_{I, J}$ we define two auxiliary functions

$$
\psi_{i, j}^{1}=\frac{1}{4}\left(\phi_{I+i, J+j}+\phi_{I-i, J-j}-\phi_{I+i, J-j}-\phi_{I-i, J+j}\right),
$$

and

$$
\psi_{i, j}^{2}=\frac{1}{4}\left(\phi_{I+i, J+j}+\phi_{I-i, J-j}-\phi_{I+j, J+i}-\phi_{I-j, J-i}\right) .
$$

By (7.9), Theorem 7.1 and (7.21) we deduce

$$
\begin{aligned}
& \left|\Delta \psi_{i, j}^{1}\right| \leqq \delta, \quad\left|\Delta \psi_{i, j}^{2}\right| \leqq \delta, \quad-\infty<i, j<\infty, \\
& \left|\partial_{y} \psi_{i, j}^{1}\right| \leqq b \delta, \quad\left|\partial_{\nu} \psi_{i, j}^{2}\right| \leqq \frac{1}{2}(a+b) \delta, \quad-\infty<i, j<\infty,
\end{aligned}
$$

and, clearly,

$$
\psi_{i, 0}^{1}=\psi_{0, j}^{1}=\psi_{i, i}^{2}=\psi_{i,-i}^{2}=0, \quad-\infty<i, j<\infty .
$$

Let $S$ be a square with the net point $P_{I, J}$ at its center and $2 b$ the length of its sides. From (7.29) and (7.30) we easily derive

$$
\psi^{1}(\dot{S}) \leqq b^{2} \delta, \quad \psi^{2}(\dot{S}) \leqq \frac{1}{2} b(a+b) \delta,
$$

which, by Theorem 3.1 and (7.28), involves estimates (7.24) and (7.25) at the arbitrary point $P_{I, J}$.

8. Remark on Noncentral Difference-Quotients. In Sections 2 and 7 we have defined $\partial_{x}, \partial_{y}, \partial_{x y}$ and $\partial_{n}$ by central-difference expressions. This is not necessary. In fact, the theorems of Sections $3,4,5,6$ and 7 remain practically the same when we replace these difference-quotients by the "forward" expressions

$$
\begin{aligned}
\partial_{x}{ }^{+} \phi_{i, j} & =(1 / h)\left(\phi_{i+1, j}-\phi_{i, j}\right), \\
\partial_{y}{ }^{+} \phi_{i, j} & =(1 / h)\left(\phi_{i, j+1}-\phi_{i, j}\right), \\
\partial_{x y}^{+} \phi_{i, j} & =\left(1 / h^{2}\right)\left(\phi_{i+1, j+1}+\phi_{i, j}-\phi_{i+1, j}-\phi_{i, j+1}\right), \\
\partial_{n}{ }^{+} \phi(P) & =(1 / h)[\phi(P)-\phi(\widehat{P})],
\end{aligned}
$$$$
P \in \dot{R},
$$

where $\hat{P}$ is defined as in Section 7 . The modifications of the theorems and their proofs are simple. They all use, instead of Lemma 2.2, the following generalization. 
Lemma 8.1. If $R$ satisfies (2.1), $\phi$ and $\psi$ are bounded and

$$
\Delta \phi_{i, j} \leqq \Delta \psi_{i, j},
$$$$
\left(x_{i}, y_{j}\right) \in R,
$$

and if the boundary $\dot{R}$ is the union of two disjoint sets

$$
\dot{R}=\ddot{R}+\dddot{R}
$$

such that

$$
\phi_{i, j} \geqq \psi_{i, j}, \quad\left(x_{i}, y_{j}\right) \in \ddot{R},
$$

and

$$
\phi(P)+\phi(\hat{P}) \geqq \psi(P)+\psi(\hat{P}),
$$

$$
P \in \dddot{R}
$$

then

$$
\phi_{i, j} \geqq \psi_{i, j},
$$$$
\left(x_{i}, y_{j}\right) \in R \text {. }
$$

Proof. Because of linearity, it suffices to treat the case $\psi \equiv 0$. This can be proved in the same way as Lemma 2.1 , with an obvious modification.

With this lemma replacing Lemma 2.2 , all the proofs of estimates for $\partial^{+}$remain essentially the same as those for $\partial$. The theorems concerning interior estimates need be modified so that the estimated difference-quotient is evaluated at the center of the square in the theorem. For example, Lemma 3.4 would now be replaced by

Lemma 8.2. Denote

$$
\bar{R}_{b b}^{+}=\left\{\left(x_{i}, y_{j}\right) \mid-b+h \leqq x_{i} \leqq b,-b+h \leqq y_{j} \leqq b\right\} .
$$

If

$$
\phi\left(\dot{R}_{b b}^{+}\right)=0
$$

and

$$
\left|\Delta \phi\left(R_{b b}^{+}\right)\right| \leqq \delta
$$

then

$$
\left|\partial_{x y}^{+} \phi_{0,0}\right| \leqq\left(\frac{4}{3} \log (b / h)\right) \delta .
$$

Proof. We define the auxiliary doubly-antisymmetric function

$$
\psi_{i, j}=\frac{1}{4}\left(\phi_{i, j}+\phi_{-i+1,-j+1}-\phi_{i,-j+1}-\phi_{-i+1, j}\right) .
$$

This function clearly satisfies

$$
\begin{gathered}
\psi_{0, j}+\psi_{1, j}=0, \quad \psi_{i, 0}+\psi_{i, 1}=0, \quad \psi\left(\dot{R}_{b b}^{+}\right)=0, \\
\left|\Delta \psi\left(R_{b b}^{+}\right)\right| \leqq \delta
\end{gathered}
$$

and

$$
\psi_{1,1}=\frac{1}{4} h^{2} \partial_{x y}^{+} \phi_{0,0} .
$$

To estimate $\psi$ we again introduce the comparison function

$$
\chi(x, y)=x y \log [2 b /(|x|+|y|)]
$$


which, in virtue of (8.12), (8.13) and Lemma 8.1, here gives

$$
\left.\left|\psi_{i, j}\right| \leqq \frac{2}{3} \delta \chi\left(x_{i}, y_{j}\right)-\frac{1}{2} \chi(h, h)\right], \quad\left(x_{i}, y_{j}\right) \in R_{A G O F} .
$$

In particular

$$
\left|\psi_{1,1}\right| \leqq \frac{1}{3} \delta \chi(h, h)=\frac{1}{3} \delta h^{2} \log (b / h),
$$

so that, by (8.14), our lemma is proved.

Similar modifications in lemmas, theorems and proofs are easily carried out throughout Sections 3, 4, 5, 6 and 7, to yield theorems for the noncentral difference quotients. In the case of the Neumann problem, the reflections about the edges (7.5)-(7.8) are replaced by reflections about interior lines parallel to, and at a distance $h / 2$ from, the edges.

Courant Institute of Mathematical Sciences

New York University

New York, New York

1. S. Agmon, A. Douglis \& L. Nirenberg, "Estimates near the boundary for solution s of elliptic partial differential equations satisfying general boundary conditions. I," Comm. Pure A ppl. Math., v. 12, 1959, pp. 623-727. MR 23 \#A2610.

2. S. Agmon, A. Douglis \& L. NirenBerg, "Estimates near the boundary for solutions of elliptic partial differential equations satisfying general boundary conditions. II," Comm. Pure A ppl. Math., v. 17, 1964, pp. 35-92. MR 28 \#5252.

3. D. F. De Santo \& H. B. Keller, "Numerical studies of transition from laminar to turbulent flow over a flat plate," J. Soc. Indust. Appl. Math., v. 10, 1962, pp. 569-595. MR 28 $\# 800$.

4. A. Douglis \& L. Nirenberg, "Interior estimates for elliptic systems of partial differential equations," Comm. Pure Appl. Math., v. 8, 1955, pp. 503-538. MR 17, 743.

5. G. E. Forsythe \& W. R. Wasow, Finite-Difference Methods for Partial Differential Equations, Wiley, New York, 1960. MR 23 \#B3156.

6. S. GerschGorin, "Fehlerabschätzung für das Differenzenverfahren zur Lösung partieller Differentialgleichungen," Z. Angew. Math. Mech., v. 10, 1930, pp. 373-382.

7. C. Mrranda, Equazioni alle Derivate Parziali di Tipo Ellitico, Springer, Berlin, 1955. MR 19, 421 .

8. H. Montvila, On the Convergence of the Numerical Solution for a Certain Partial Differential Equation of Third Order, New York University, Institute of Math. Sci., IMM-NYU 256, 1958.

9. J. Schauder, "Über lineare elliptische Differentialgleichungen zweiter Ordnung," Math. Z., v. 38,1934 , pp. 257-282.

10. J. SchaUder, "Numerische Abschätzungen in elliptischen linearen Differentialgleichungen," Studia Math., v. 5, 1934, pp. 34-42. 